\title{
Computational Fluid Dynamic Models as Tools to Predict Aerosol distribution in Tracheobronchial Airways
}

\author{
Claudia Atzeni ${ }^{1}$, Gianluca Lesma ${ }^{1}$, Gabriele Dubini ${ }^{1}$, Maurizio Masi ${ }^{2}$, Filippo Rossi ${ }^{2}$ and Elena Bianchi $^{1 *}$ \\ ${ }^{1}$ Laboratory of Biological Structures Mechanics (LaBS), Department of Chemistry, Materials and Chemical Engineering “Giulio Natta”, Politecnico di \\ Milano, Italy \\ ${ }^{2}$ Department of Chemistry, Materials and Chemical Engineering “Giulio Natta", Politecnico di Milano, Italy
}

Submission: June 01, 2020; Published: July 06, 2020

*Corresponding author: Elena Bianchi, Laboratory of Biological Structures Mechanics (LaBS), Department of Chemistry, Materials and Chemical Engineering “Giulio Natta”, Politecnico di Milano, Italy

Abstract

Aerosol and pollutants, in form of particulates 5 - 8 um in main size face every day our respiratory system as natural suspension in air or forced to be inhaled as a coadjutant in a medical therapy for respiratory diseases. This inhalation happens in children to elderly, women and men, healthy or sick and disable people. In this paper we analyzed the inhalation of aerosol in conditions assimilable to the thermal therapy, in a model of patient representing an adult man. We use a computational fluid dynamic 3D model to compute and visualize the trajectories of aerosol down to the sixth generation of bronchi, in a dynamic condition set as breath cycle at rest. Results, compared to a set of milestones experimental studies published in literature, allow the comprehension of particles behavior during the inhalation from mouth to bronchi sixth generation, the visualization of jets, recirculation and deposition, in a characteristic geometrical model including rings. Numerical and graphical results, usable from researchers, technicians and medical doctors, may enable the design of medical devices and protocols to make the inhalations more effective in all the user's population.

Keywords: Respiratory particle dynamics; Particle deposition; Bifurcation models; Lagrangian particle tracking; Pharmaceutical aerosol; Drug delivery

\section{Introduction}

Understanding particle deposition is important in risk assessment for toxic air pollutants as well as in evaluation of the efficacy of therapeutic aerosols. The complex lung morphometry, the cyclic and unsteady nature of the respiratory flow, air and particle properties have major effects on trajectories and deposition of inhaled particles, [1]. The use of computational fluid dynamic (CFD) tools allows the robust description and prediction of transport and deposition of particles in a defined 3D structure, based on mathematical models. The analyses and the visualization of complex phenomena in a 3D accessible format is a determining tool in making clinical decisions, designing biomedical devices and clinical protocol, and in explaining phenomena for educational purposes. The first step of the computational approach is the definition of a geometrical model. Many models have been created to investigate airflow patterns and particle transport in the respiratory airways through experimental and computational approaches, [2]. According to Walenga et al., [3], such models can be categorized as simplified, patient-specific and characteristic models. Simplified models are geometrically similar to the respiratory airways but miss geometric features that could affect quantitative outputs. Patient-specific models are obtained as reconstructions from in-vivo images and they are able to correctly reproduce all the phenomena occurring in an individual subject. A characteristic model, as the one described in this paper, reproduces all geometrical features of an average subject while neglecting unnecessary patient-specific details that could decrease the predictive efficiency of the model. Considering the domain extension, these models can be further categorized as single [4] double [5] or multiple [6,7] bifurcation models, complete tracheobronchial (TB) tree models, [8-10], upper airways models [11-13] and complete airways models [3,14-20].

The most used simplified model was created by Weibel et al. and consists of 23 generations of symmetric bifurcating airways [21], defined by their lengths, diameters and branching angles between parent and daughter branches. Based on Weibel data, Balàshàzy et al. [4] proposed a model of realistic asymmetric bifurcation where 
the parent and daughter tubes do not lie on the same plane and are delimited by a transition zone, which contains the two curved portions of daughter tubes [4,22]. Similarly, Heistracher et al., [23], defined a mathematical method to generate a 3D asymmetric bifurcation model with a smooth surface through the definition of the radius of curvature and the carinal ridge. In order to achieve a precise model of the human TB tree, in-vitro preparation of lung casts taken from cadavers can be combined with high resolution computed tomography imaging (CT) technique. The most widely used model is the digital reference model proposed by Schmidt et al., [8], which contains 1453 bronchi extending down to the $17^{\text {th }}$ generation. With regard to the upper airways, a number of studies tried to assess the role of upper morphometry on particle DE. Using measurements from CT scans, magnetic resonance imaging (MRI) and direct observation of patients, they describe idealized models of the extra thoracic airways with simple geometric features, reproducing mouth, pharynx, larynx and trachea. Cheng at al., [11], investigated characteristic shapes, perimeters and cross-sectional areas (CSA) of different sections of human upper airways extending from the oral cavity to the upper trachea. CSA varies several times along the model, with a minimum of $0.84 \mathrm{~cm}^{2}$ in proximity of the glottis. Similarly, Stapleton et al., [12], created an average model of extra thoracic airways where both pharynx and glottis are simplified as elliptical tubes with CSA equal to 2.98 $\mathrm{cm}^{2}$ and $0.95 \mathrm{~cm}^{2}$, respectively. Starting from Cheng model [11], Xi et al. [13], introduced realistic features such as the physiological airway curvature, a half-mouth opening, a triangular-shaped glottis and the $17^{\circ}$ dorsal angle. A model involving the all pathway from the first bifurcation to the sub-acinus unit, by means decomposition and reduction of complexities of the TB, is the one presented by Koullapis [24]. Recently, complete models of human airways comprising both upper airways and TB tree down to the $6^{\text {th }}-7^{\text {th }},[3,14,15,18,19]$, or to the $17^{\text {th }},[25]$ generations have been realized. On each of these reconstructed 3D airways model it was possible to applied mathematical and numerical methods and perform CFD simulations of airflows in flowing and depositing particles to target anatomical zones. The 3D model of interest has to be discretized by the definition of a grid, whose quality and topology influence the quality and the robustness of the resulting data. Flow is computed by means of numerical models of the Navier-Stokes equation. The effects of air fluid dynamics and particle properties can usually be assessed considering various combinations of Reynolds and Stokes numbers, [3,15,20,26-30]. Reynolds is a dimensionless number, ratio between inertial and viscous forces, and is it used to define the laminar/turbulent regime of flow in the model.

Stokes is another dimensionless number defined as the ratio between the characteristic response time of the fluid and the characteristic response time of the particle, and it is used to characterized the ability of particles to follow the fluid streamlines [31]. The transport and deposition of particles, intended to represent pollution or aerosol particles, are investigated by means of dedicated numerical models, interacting or consequent to the airflow models. Particle deposition is commonly evaluated in terms of particle deposition efficiency (DE), defined as the dimensionless ratio of the number of particles deposited in a given region to the total number entering the region $[5,7,12,13,17,27,28]$.

Accurate steady state simulations, with a constant flow rate at the inlet, defined as the representative condition in airways leading to particle deposition, were described in the several works of the scientific literature. Comer et al., [5], numerically simulated deposition of inhaled 3-7 $\mu$ m particles in a 3D double bifurcation model, assuming a steady, laminar and constantproperty airflow. They observed a variation in deposition patterns with the bifurcation level and orientation. The distribution was symmetric near the carina of the $1^{\text {st }}$ bifurcation, while it appears very asymmetric at the $2^{\text {nd }}$ generation walls. Similarly, Zhang et al., [32], extended this analysis down to the $3^{\text {rd }}$ bifurcation simulating spherical non-interacting particles. Gemci et al., [9], performed simulations by CFD to investigate airflow pattern in Schmidt [8], $17^{\text {th }}$ generation model. A constant airflow rate of $28.3 \mathrm{~L} /$ min was imposed to simulate the turbulent regime using a large eddy simulation (LES) turbulence algorithm. With an airflow rate ranging from sedentary to normal breathing conditions, they observed the development of secondary patterns in the 1st generation well as the increase of the viscous pressure drop from $6 \mathrm{~Pa}$ at $6 \mathrm{~L} / \mathrm{min}$ to $54 \mathrm{~Pa}$ at $30 \mathrm{~L} / \mathrm{min}$ [9] Xi et al. [13], imposed a constant airflow rate of $30 \mathrm{~L} / \mathrm{min}$ to the realistic featured model, to simulate a turbulent airflow using the low Reynolds number (LRN) k- $\varepsilon$ model. A laryngeal jet appeared upstream the glottis region due to the reduced CSA and produced recirculation zones near the trachea. It was observed that the laryngeal jet combined with a physiological trachea dorsal angle enhanced particle deposition around the glottis and the upper trachea.

Lambert et al. [15], investigated regional deposition of 2.5 - $30 \mu \mathrm{m}$ particles in a CT-based human airway model down to the $7^{\text {th }}$ generation. The turbulent flow in the upper respiratory tract was simulated with large eddy simulation (LES) imposing a steady-state parabolic airflow of $20 \mathrm{~L} / \mathrm{min}$ at the oral inlet. Particle deposition increased with increasing particle size and in proximity of bifurcations, due to the inertial effects. The filtering effect of the oral cavity was very pronounced for $30 \mu \mathrm{m}$ particles, while DE of $10 \mu \mathrm{m}$ particles was more uniform in spite of high concentration in larynx. They also highlighted that the left lung received a greater portion of the particles with respect to the right lung, in spite of the greater flow ventilation in this region. Similarly, Rahimi-Gorji et al. [19], analysed airflow and particle deposition under various breathing conditions in a realistic airway model down to the $6^{\text {th }}$ bronchus generation, obtained as a reconstruction from CT scans. Steady-state CFD simulation were performed to solve the flow field while spherical and inert particle deposition was simulated using the discrete phase model (DPM). At higher flow rates $(>30 \mathrm{~L} / \mathrm{min}$ ) the region between pharynx and larynx showed maximum velocity near the outer wall due to centrifugal forces, thus trapping large diameter particles. On the other side, in a laminar regime $(<15 \mathrm{~L} / \mathrm{min})$, the lower inertial forces directed 
more particles deeper up to the $4^{\text {th }} 5^{\text {th }}$ generations. In Kim et al. [7], the results of simulation on a new realistic parametrically controlled airway model, down to the $11^{\text {th }}$ generation, were compared to Weibel model, finding a good agreement. In a more recent times, the advancement in the simulation tools and the increasing of the IT calculation capacity have led to the design of more complex and ambitious models, involving a deeper featuring of the anatomical and respiratory details. The lung model of Elcner et al., [14], contains realistic geometries for the throat, the trachea and the TB tree up to the $4^{\text {th }}$ generations. Data of upper airways were acquired from CT scans of living subjects, while the reconstruction of TB tree was based on Schmidt data. Experiments and transient CFD simulations were carried out to reproduce a complete sinusoidal breathing cycle under both sedentary (7.5 L/min) and deep breath ( $15 \mathrm{~L} / \mathrm{min})$ regime. A laryngeal jet clearly formed and evolved during the breathing cycle and its effect propagated downstream the trachea with the formation of separation zones in both the left and right main bronchi. Calmet et al. [18], performed large-scale CFD simulation on a highly detailed geometry of lung down to the $4^{\text {th }}$ bifurcation in order to explore the airflow dynamics during a rapid inhalation cycle. A dominant turbulent flow was observed along the descending airways due to the strong laryngeal jet formed, which quickly dissipated with the breakup of vortex structures. They found out that nasal and supraglottic regions were the dominant sites of turbulence production and were thus characterized by the highest values of wall shear stress. Walenga et al. [3], compared data obtained from simplified and realistic models of TB tree down to the $3^{\text {rd }}$ generation with the empirical data of Zhou et al. [27], to quantify the influence of geometrical features (i.e. tracheal curvature, main bronchi curvature and irregular cross sections) on pharmaceutical aerosol deposition. They found that accurate tracheal features enhanced regional deposition and shifted particle patterns towards left walls. Realistic CSAs and bifurcation curvature increased DE in the TB tree [3]. Their results showed that the respiratory air flow characterized in the lung is nonsymmetrical while the pattern of flow field was similar.

Das et al. [20], used an idealized, anatomically faithful upper airway geometry, where simulations are function of age, from a five-year-old to an adult. The results of the comparison between a Dry Powder Inhalers (DPI) and

nebulizer inhalation performance were collected in a dimensionless curve governing deposition in the airways via Stokes number. A patient-specific model was then simulated by Farghadan et al. [10], within a breathing cycle, and the use of Finite-time Lyapunov exponent (FTLE) was involved to compute the Lagrangian topological maps that define the destination of particles at the trachea. Tian et al. [16], compared steady state and transition simulations of particles deposition in a characteristic patient model, by means of a Stochastic individual path (SIP) model in branches deeper than TB4.
CFD results were compared for validation to experimental data on total and regional DE. Experimental data were obtained from both human volunteers and airway hollow casts, [27,28,30,3337]. In-vivo particle deposition in the mouth, pharynx and larynx can be tracked using radio-labelled particles injected from the mouth, while the amount of deposited particles in a cast can be evaluate using radiometric counting or chemical analyses [1]. For example, Chan and Lippmann [30], studied the regional DE in a hollow cast of the human larynx and TB tree extending down to the $6^{\text {th }}$ generations and in 26 human volunteers in vivo, finding a linear dependence of particle DE on Stokes number for aerosols with aerodynamic diameters greater than $2 \mu \mathrm{m}$.

Similarly, Cheng et al. [28,33], investigated the effects of particle size and breathing conditions on DE in a human oral airway replica. They observed that DE increased with higher flow rates and particle diameters, suggesting that impaction is the dominant deposition mechanism. Furthermore, by comparing their results with in-vivo deposition data [30,37], they defined a relationship between DE and the impaction parameter IP, defined as:

$$
I P=\rho d_{p}^{2} Q
$$

where $\rho$ is the density of the particle, $d p$ is the aerodynamic diameter and Q is the flow rate [28]. Regional DE in a TB tree replica was carefully studied by Zhou et al. [27], through the injection of fluorescent 0.93-30 $\mu \mathrm{m}$ particles at constant flow rates of 15 , 30 and $60 \mathrm{~L} / \mathrm{min}$. They observed a relationship between DE and Stokes numbers, bifurcation angles and diameters and provided an empirical model to estimate DE in each generation.

Many partial or complete model of the human airways were created to investigate airflow pattern and particle DE under various breathing conditions. Airflow pattern was widely analyzed under both constant flow rates, $[5,9,13,15,25]$, and transient breathing cycle $[14,18,38]$, while most of particles analyses found in the literature were performed under constant airflow conditions. Gurman et al. [36], suggest that this approximation can represent large particle cases pretty well while it underestimates total DE of smaller particles.

Only a few recent works analyzed the behavior of nanometric and micrometric particles during a complete breath cycle $[10,16,24,38]$ and under different breathing conditions (sedentary, light and heavy breathing). The focus of the present research is to create a characteristic model of human airways, belonging an average adult male, in order to investigate therapeutic aerosol DE reproducing sedentary breathing conditions, including the transient behavior of a physiologically breathing cycle. A deep analysis of the anatomical features described in literature suggested the involvement of a special strategy to design the geometry of bronchi around the bifurcation and the introduction of the cartilaginous rings in the reconstruction of the trachea. Rings are usually described as structural components of the 
tracheal wall but their presence modifies the appearance of the tracheal wall as sub- millimetric features [16] that may have a role in the formation of boundary layer of flow and in the deposition of particles on the surfaces, consistently with in-vitro observations of Russo et al. [39].

\section{Materials and Methods}

\section{Anatomical model}

The 3D airway model contains a characteristic geometry of the oral cavity, throat and TB tree down to the 6th

generations Figure 1(a-b), including physiological bifurcations and cartilaginous rings. The reconstruction of the TB tree was based on coordinates and dimensions of the Schmidt digital model [8], (SM.1), and obtained with the computer-aided design (CAD) software Solid works 16 (Dassault Systèmes Solid works Corp., Waltham, MA USA). A special attention was given to the design of branches sizes and bifurcations. The relationship between parent (P) and daughter branches (A, B) follows Eq. (1):

$$
d_{P}^{x n}=d_{A}^{x n}+d_{B}^{x n}
$$

where $d_{P}^{x n}$ is the parent branch diameter, $d_{A}^{x n}$ and $d_{B}^{x n}$ are the daughter ones and $x$ is the exponent which determines the flow conditions in the $n-t h$ bifurcation.

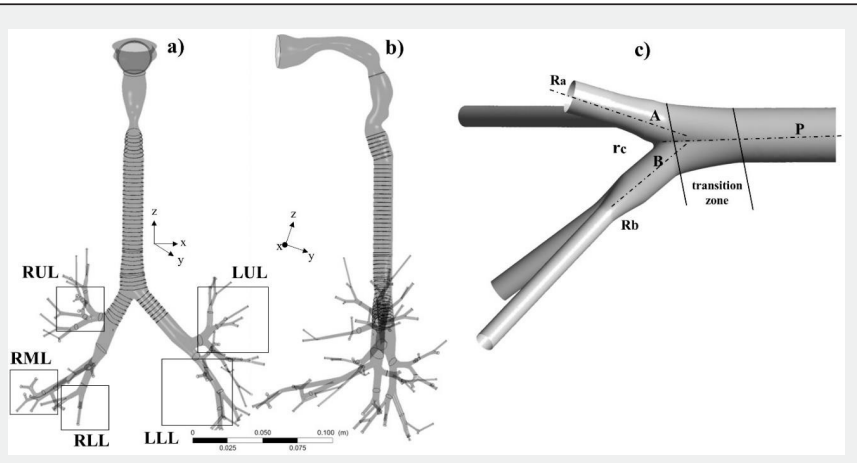

Figure 1: Frontal (a) and lateral (b) view of the 3D realistic airway tree created and the correspondent lobes: right-upper (RUL), right-medium (RML), right-lower (RLL), left-upper (LUL) and left-lower (LLL). (c) Model of physiologically realistic bifurcation based on Heistracher et al. [22]; $r_{c}$ is the carinal radius of curvature, $R_{A}$ and $R_{B}$ are the daughter radii and $c c$ is the ratio of carinal curvature ratio.

To reproduce physiologically characteristic bifurcations Figure 1c, every parent segment was split into two daughter tubes with a transitional zone starting from the $80 \%$ of its axial length. Then, each transition zone was smoothed defining the characteristic carinal radius of curvature $r c$ through the equation:

$$
r_{c}=\left(R_{A}+R_{B}\right) c_{c}
$$

where $R_{A}$ and $R_{B}$ are the daughter radii and $c c$ is the ratio of carinal curvature ratio equal to 0.1 , [23]. The tracheal section was modelled as a circular tube with a diameter equal to 1.56 $\mathrm{cm}$, cut by a posterior plane at $0.65 \mathrm{~cm}$ from the centre [40] thus reproducing the tracheal typical C-shape due to the presence of the pars membranacea [41]. As shown in Figure 2(a-c), tracheal and main bronchi walls were enriched with cartilaginous rings, whose dimensions are consistent with in-vitro observations of Russo et al. [39].The rings arrangement was made regularly perpendicular to the axial branch direction, while the rings orientation was adapted in the transitional zones to maintain their parallelism with the curvature radii.

A characteristic model of extra thoracic airways was created by shaping the oral cavity, pharynx and larynx as elliptical tubes Figure $2 \mathrm{~b}$. Geometrical parameters of each section were extracted from Cheng's silicone model, [28]. Then hydraulic diameters and cross-sectional areas (CSA) were scaled in order to fit Schmidt realistic model of TB tree. In addition, the trachea was oriented at an angle $\alpha=17^{\circ}$ from the vertical axis to match CT-scan analyses by Xi and Longest and the anatomical curvature of the throat was reproduced by imposing a curvature radius $r=3.27 \mathrm{~cm}$ [13].

\section{Grid generation}

The 3-D characteristic airway model was divided into 24 bodies according to size and anatomical region Figure 2a : oral cavity and pharynx, larynx, trachea, main bronchi bifurcation $\left(1^{\text {st }}\right.$ bif.), second ( $2^{\text {nd }}$ bif.) and third ( $3^{\text {rd }}$ bif.) generations. There are 64 peripheral small airways. Ansys ICEM CFD software (Ansys Inc., Canonsburg, PA, USA) was employed to discretize the model with tetrahedral volume elements, triangular superficial elements and a multi-layer prism mesh to solve the high velocity gradient expected at the upper airways and larynx walls Figure 3. The best size of the grid to solve this problem (tradeoff between accuracy of results and calculation time) was selected by a sensitivity analysis (SM2) and results in a mesh of 9,826,620 tetrahedral elements and three boundary layers of hexahedral ones.

\section{Numerical solutions and settings}

Airflow: Numerical simulations were performed with the commercial CFD code Fluent 16.0 (ANSYS, Inc., Canonsburg, PA, USA) on a Dell T630 Workstation with 64Gb RAM and two $1.8 \mathrm{GHz}$ CPUs. Air was assumed to be an incompressible and Newtonian fluid with constant density $\rho=1.225 \mathrm{~kg} / \mathrm{m} 3$ and viscosity $\mu=$ 
$1.789410-5 \mathrm{~kg} / \mathrm{m}^{-s}$. The continuity and Navier-Stokes equations (Eqs. 3 and 4) for the airflow phase were discretized with second order accuracy in time and space under both steady-state and transient conditions. The Green-Gauss Node-Based algorithm was utilized for pressure-velocity coupling:

$$
\begin{gathered}
\frac{\partial \rho}{\partial t}+\nabla(\rho \vec{u})=0 \\
\rho\left\{\frac{\partial \vec{u}}{\partial t}+(\vec{u} . \nabla) \vec{u}\right\}=\nabla \rho+\mu \nabla^{2} \vec{u}+\rho \vec{f}
\end{gathered}
$$

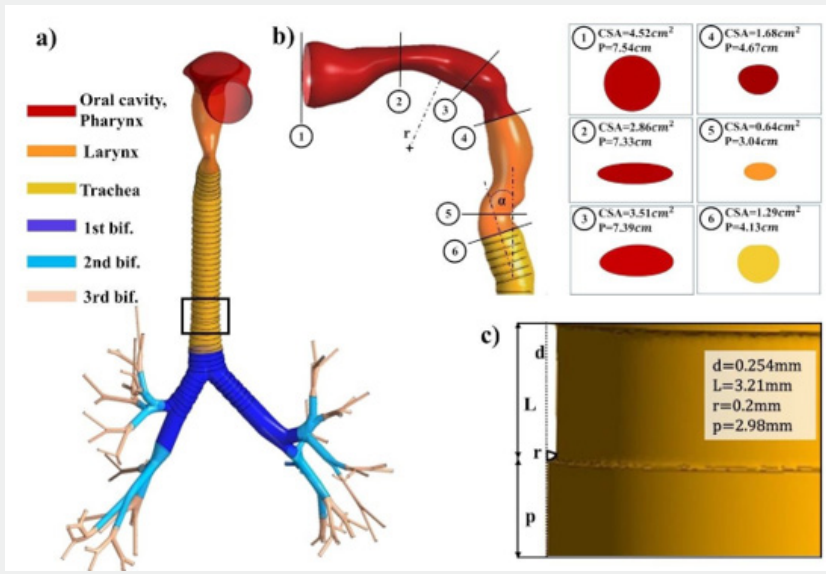

Figure 2: (a) Different regions of the respiratory system. (b) Critical cross-sectional areas and perimeters of the upper airways model; $r$ is the anatomical curvature radius of the throat, $\alpha$ is the orientation of the trachea with respect to the vertical axis, [13]. (c) Dimensions of the cartilaginous rings, based on in-vitro observations of Russo et al., [40]; $p$ and $L$ are the step and the width, respectively, $d$ is the depth of the ring and $r$ is the fillet radius.

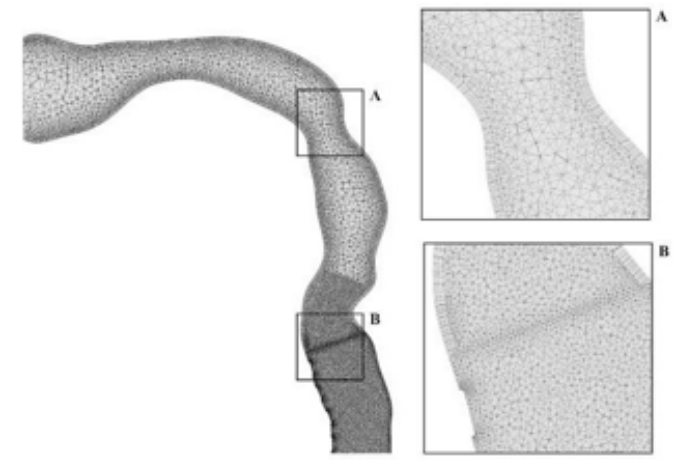

Figure 3: Representative computational grid of the model. Section A shows the prismatic layers created near the larynx wall; Section B shows the finest tetrahedral element of the tracheal mesh.

Transient CFD simulations were performed to reproduce a complete sedentary breathing cycle. Such a cycle was modelled with a sinusoidal curve 4-second-long, with inhalation and exhalation phases of the same duration [14], Airflow had mean and peak values equal to $6 \mathrm{~L} / \mathrm{min}$ and $9.42 \mathrm{~L} / \mathrm{min}$, respectively. A user-defined function (UDF) was implemented to impose a time-dependent, sinusoidal velocity profile at the $i^{\text {th }}$ outlet of the model, through the formula:

$$
u_{i}(t)=u_{i, \max } \operatorname{Sin}\left(\frac{2 \pi t}{T}\right)
$$

where $u_{i, \max }$ is the maximum velocity at the $i$-th outflow extracted from steady-state solutions (SM3), T is the breathing period and $t$ is time. No-slip boundary conditions and zero pressure were assumed at airway walls and at the oral inlet, respectively.

Particles: Particle transport equations were solved using the discrete phase model (DPM) available in Fluent (ANSYS, Inc., Canonsburg, PA, USA), in combination with user-defined injection patterns and boundary conditions. DPM model (SM4) tracks individual particles as they move through the flow using a Lagrangian approach. The Navier-Stokes equations for the 
continuous and discrete phases were solved separately in the steady-state condition, while they were solved together in the transient regime. Particle deposition is usually assessed as a function of particle Stokes number St:

$$
S t=\frac{\rho p d_{p}^{2} u c_{c}}{36 \mu R_{0}}
$$

where $\mathrm{R}_{0}$ and $\mathrm{U}$ are the average radius and the mean velocity of the airflow in the parent branch, respectively and $\mathrm{C}_{\mathrm{c}}$ is the Cunningham slip correction factor defined by Hinds et al. [31]. St is typically considered to express the ratio between the particle stopping distance and a characteristic dimension of an obstacle. Thus at large Stokes numbers, particles may deviate from fluid streamlines and impact on obstacle surface, while at small numbers they tend to follow fluid streamlines [15,31]. The particles simulated in our analyses had constant diameters $\left(3 \leq d_{p} \leq 25\right.$ $\mu \mathrm{m})$, which is the typical range of aerosol treatment. Density and viscosity were set equal to water liquid ones, thus $\rho=998.2 \mathrm{~kg} / \mathrm{m}^{3}$ and viscosity $\mu=0.001 \mathrm{~kg} / \mathrm{m}^{\text {s }}$ and inert behavior was considered. $C_{c}$ was set equal to 1 , in accordance with observations reported in Hinds et al. [31] and Tu et al. [42]. The injection pattern consisted of 2,720 particles homogeneously distributed on the oral surface, forming a disk with a diameter equal to $2.16 \mathrm{~cm}$ at a constant distance from the oral boundary. The boundary conditions for the equations governing particle motion included zero initial velocity, deposition when the particle centre reaches the wall and escape conditions at the 64 outlets. Four different diameters equal to 3, 7, 10 and $25 \mu \mathrm{m}$ were considered to assess the effect of particle size on DE under a constant airflow rate $(6 \mathrm{~L} / \mathrm{min})$. Numerical predictions of total and regional DE were compared with both empirical formulations and experimental evidence available in the literature $[3,15,28,30,34]$. Tracheobronchial deposition data were validated with the empirical results of Zhou et al. [27], based on in vitro experiments of polystyrene latex particles. The general formulation used for DE calculation was:

$$
D E=1-\exp \left(-a^{*} S t^{b}\right)
$$

where $\mathrm{a}$ and $\mathrm{b}$ are empirical constants, which differ according to the airway of interest. Upper airways DE were

compared with the experimental evidences found in Cheng et al. [28] on oral airway silicone replicas, defining DE as a function of the impaction parameter $I P=\rho d_{p}^{2} Q$ :

$$
D E=1-\exp \left(-a^{*} I P\right)
$$

DE of $7 \mu \mathrm{m}$ particles was also analyzed under unsteady condition. The same distribution of 2,720 particles were

injected at the oral inlet every $0.2 \mathrm{~s}$ during both ascendant [at $0,0.2,0.4,0.6$ and $0.8 \mathrm{~s}$ ] and descendant parts [at 1, 1.2, 1.4, 1.6 and $1.8 \mathrm{~s}$ ] of the inhalation phase. Furthermore, an in-house MATLAB code (MATLAB, The MathWorks, Inc., Natick, MA, USA) was implemented to calculate the amount of particles exiting from the 64 outlets of the model.

\section{Results}

Results of numerical simulations are tools to visualize and describe the behavior of air and aerosol along a respiratory cycle.

\section{Air flow}

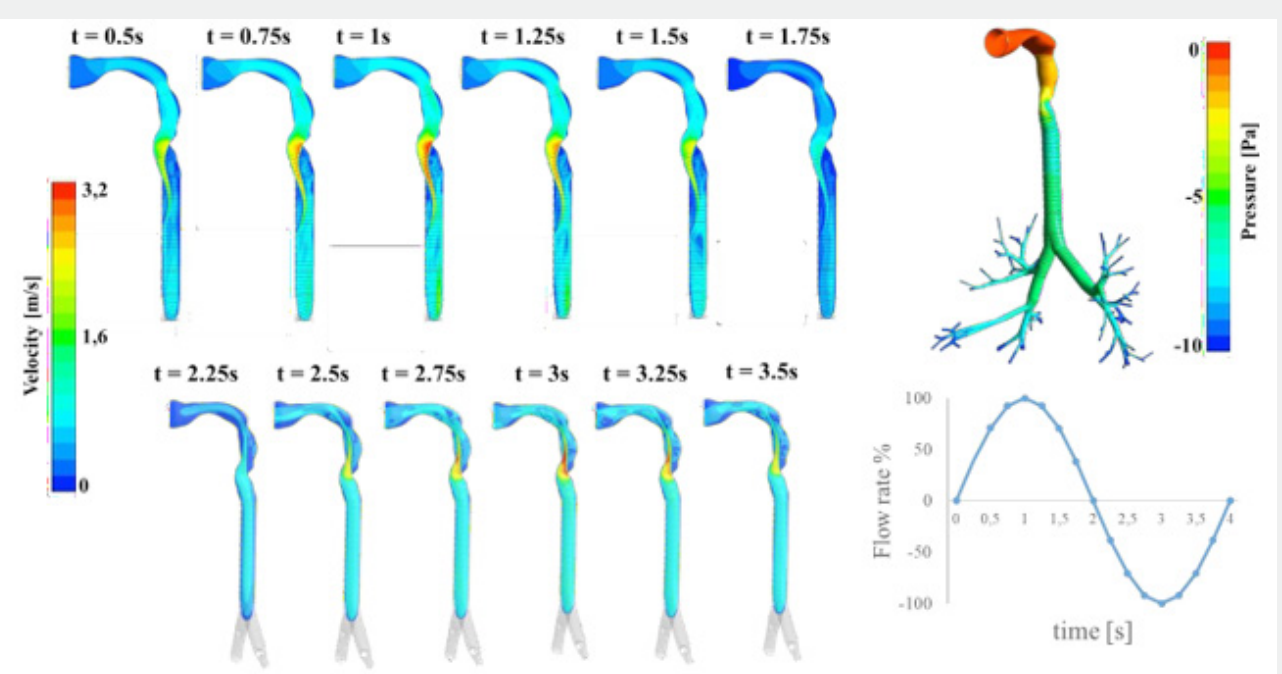

Figure 4: (a) Airflow pattern on a longitudinal plane during the sedentary breathing cycle. (b) Static pressure field along the respiratory system at the inspiration peak $(t=1 \mathrm{~s})$.

Right ventilation was quantified considering the airflow exiting the RUL (right-upper lobe), the RLL (right-lower lobe) and the RML (right-medium lobe), while left ventilation took into account the LUL (left-upper lobe) and the LLL (left-lower lobe) outflows. Reasonable consistency of the percentage ventilation with published data [43-45] was found for each of the five lung 
lobes: RUL 19\%, RLL 29\%, RML 9\%, LUL 26\% and LLL 17\%. The total left and right total ventilation values were $43 \%$ and $57 \%$, respectively. The resultant distribution is translated to the transient simulation, by means of the dedicated script. The velocity profile at each cross section varies continuously during one complete cycle of respiration, depending on cross sectional areas, the geometrical features and the velocity profile at the inlet of the region (Figure 4a) [1]. As expected, a laryngeal jet appears in the glottis region, where the noticeable constriction of the larynx forces the airflow to accelerate $[16,20]$. The strong centrifugal forces shift this high velocity region towards the posterior wall during the inhalation phase $(0-2 \mathrm{~s})$, while the peak is shifted towards the anterior walls during the exhalation phase (2-4s). Nevertheless, airflow always exhibits the characteristics of laminar or quasi-transitional flow, reaching a maximum Reynolds number equal to $\mathrm{Re}=1848$ at $\mathrm{t}=1 \mathrm{~s}$ in the glottis. The absence of a turbulent regime is reasonable considering the sedentary breathing condition simulated: turbulent phenomena appear for airflow greater than 10-12 L/min [1,9,14,25].

The static pressure distribution in Figure $4 \mathrm{~b}$ shows a pressure drop equal to $4.5 \mathrm{~Pa}$ between the tracheal inlet and the $6^{\text {th }}$ branches at the inspiratory peak, which is close to measurement by Gemci et al. [9]. The laryngeal jet effect propagates downstream the trachea with the development of secondary structures in the radial direction (Figure 5). These recirculation zones were further investigated in terms of vorticity. At $t=0.2 \mathrm{~s}$ two opposite vortices appear near the dorsal wall of section T1 (Figure 6), then they progressively develop until they reach a maximum value of vorticity equal to $500 \mathrm{~s}^{-1}$ at the inspiratory peak $(t=1 \mathrm{~s})$. Each vortex core is progressively shifted towards the anterior wall (T3) downstream of the trachea, while the intensity decreases. In the proximity of the 1st bifurcation, the main bronchi velocity profile (LMB, RMB) has the shape of a parabola with the peak near the inner walls, which is confirmed by other works $[1,9,14,46]$. Airflow pattern in downstream branches is regular and symmetric, due to the highly laminar regime of these regions.

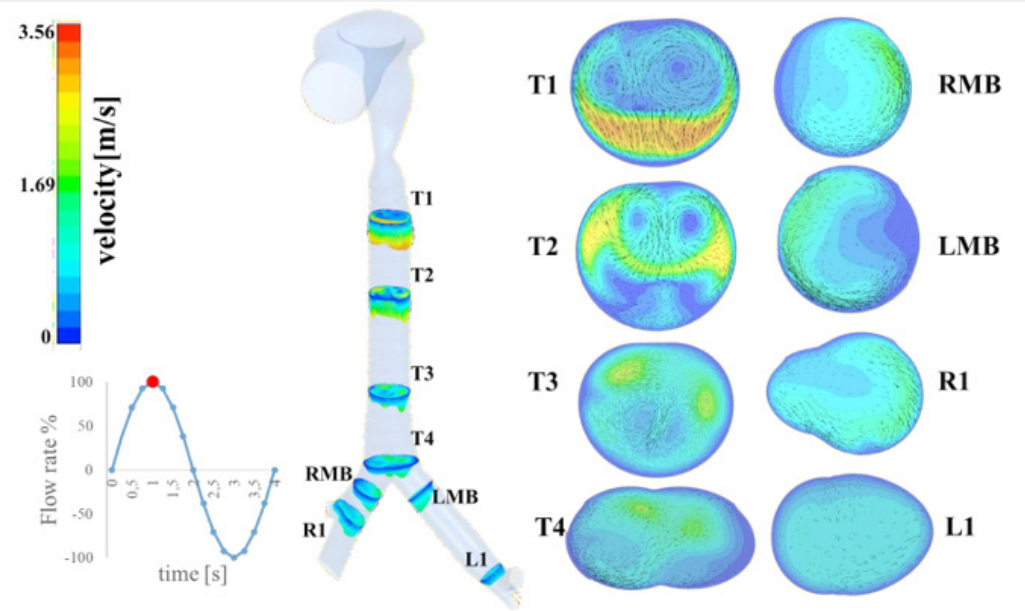

Figure 5: Development of the velocity field downstream the trachea at $t=1 \mathrm{~s}$ in terms of velocity magnitude and tangential component of the velocity vectors.

Figure 6: Time development of the vorticity field at cross section T1 during the ascendant part of the inhalation cycle $(0-1 \mathrm{~s})$. 


\section{Particulate phase}

Steady DE (eq.7) of 3-25 $\mu$ m particles in the oral cavity, larynx, trachea, 1st bifurcation and the four lobes are listed Figure 7. Increasing particle size, thus particle inertia, the filtering effect of larynx increases. DE of $7-10 \mu \mathrm{m}$ particles is more uniform although a high concentration correctly persists in the larynx [15]. Generally, the right lung receives a greater portion of the particles, which is reasonable considering the greater flow ventilation in this region and the laminar regime simulated (<10 L/min) [19].

Figure 7: Deposition efficiency (DE) of $3-25 \mu \mathrm{m}$ particles injected under steady conditions (Q=6 L/min). RUL and RLL are the right upper and lower lobes, LUL and LLL are the left upper and lower ones.

Regional deposition efficiency of 3-25 $\mu$ m particles in the oral cavity, trachea and in the $1^{\text {st }}$ and $2^{\text {nd }}$ bifurcations is shown in Figure 8, respectively. DE in the upper airways is presented as a function of the impaction parameter IP (eq.1), while the Stokes number (eq.2) better represent the DE behavior in downstream regions. DE of our characteristic model showed a similar trend as the empirical model of Zhou et al. [27] and other data found in the literature $[3,15,28,30,34]$. Good agreement between the empirical formulation and the characteristic model data was achieved with $7 \mu \mathrm{m}$ particles, especially at the 1 st and $2 \mathrm{nd}$ bifurcations.
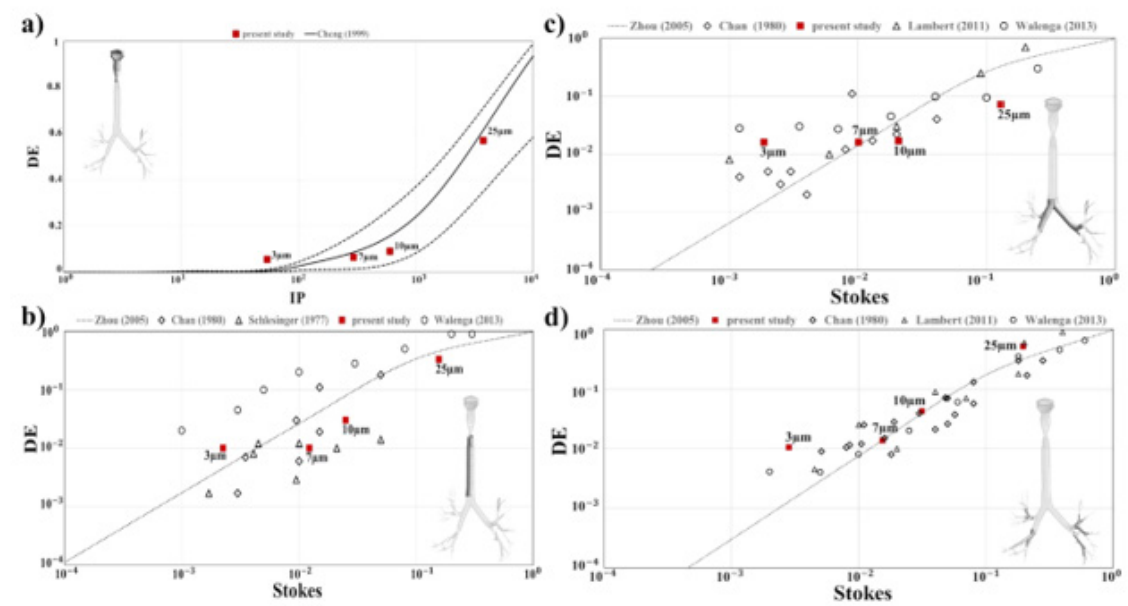

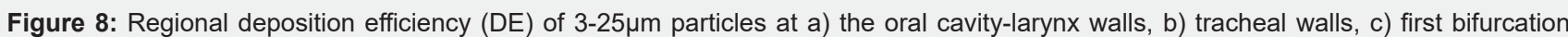
walls, d) second bifurcation walls, under steady conditions. The results are compared with the empirical formulation of Zhou et al. and other studies available in literature. 
Concerning the deposition of $7 \mu \mathrm{m}$ aerosol deposition in an unsteady simulation, total and regional DE of the airway was analyzed at various injection times Figure 9a. In the upper regions of the model (oral cavity, larynx and trachea) DE increased with increasing injection time and reached the maximum value at the inspiratory peak $t_{i n j}=1 s$. It rapidly decreased during the deceleration phase (1-2s) of the inhalation cycle. Particles mostly deposited at the larynx walls, where the effect of the laryngeal jet combined with geometrical features determined a peak value of DE equal to $15.07 \%$. At the $2^{\text {nd }}$ and $3_{\text {rd }}$ bifurcations, DE reached the maximum value at $t_{i n j}=0.6 \mathrm{~s}$, while injecting particles at $t_{i n j}=0.8 s$ seemed to be more efficient concerning the $1^{\text {st }}$ (main bronchi) bifurcation.

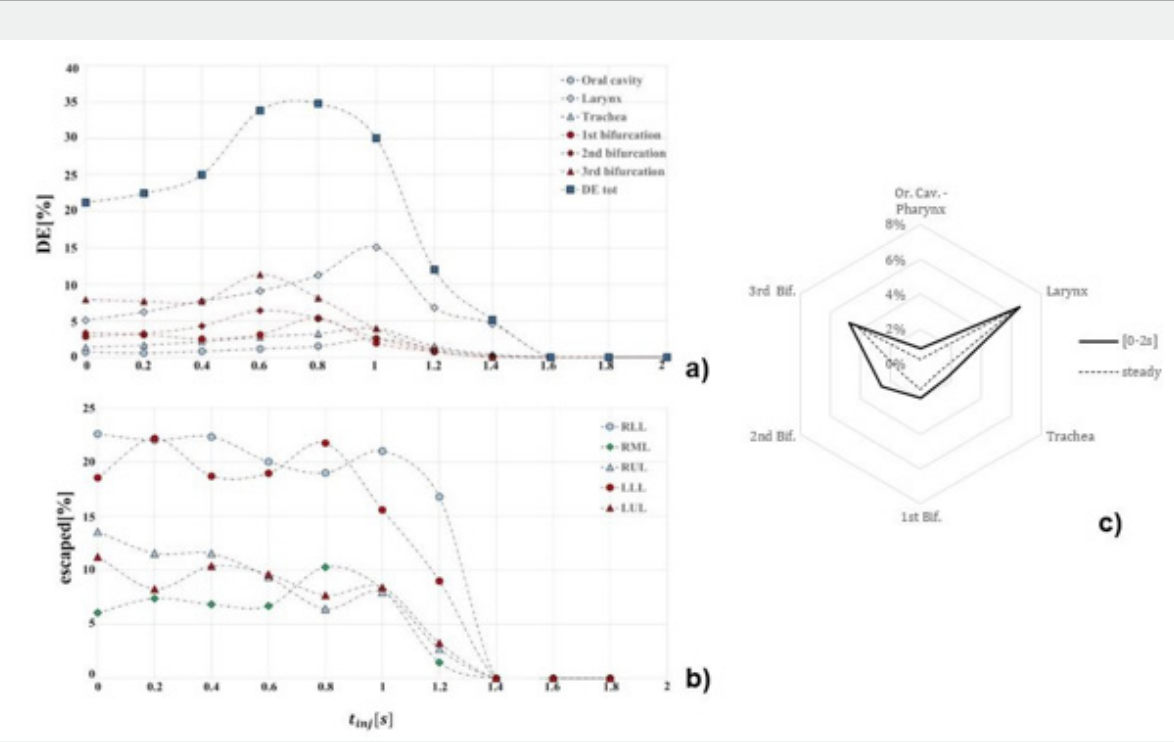

Figure 9:a) Total deposition efficiency (DE) of $7 \mu \mathrm{m}$ particles in different regions of the model for various injection times; b) percentage of particles escaped from the different lobes of the lung model for various injection times, c) total DE in steady and unsteady simulations, for different regions.

Injection at $t_{i n j}=0.8 \mathrm{~s}$ was the most efficient strategy also considering the amount of particles deposited in the whole model: total DE was equal to $34.78 \%$, which is $20.33 \%$ larger than the one observed in steady simulations. The unsteady and cyclic nature of the airflow improved TB deposition, leading to a total DE of $18,44 \%,+4 \%$ than the one observed under steady conditions; detailed deposition is reported in Figure 9c. The percentage of particles leaving the terminal branches at various injection times is presented in Figure 9b. The number of particles escaped from the lower (RLL, LLL) lobes is larger than that of the particles leaving the upper and medium lobes (RUL, RML, LUL). Maximum percentages were reached with the first and second injections at $t_{i n j}=0-0.2 \mathrm{~s}$, except for the RML lobe, where more particles left the RML when injected at $t_{i n j}=0.8 \mathrm{~s}$. On the other side, particles injected during the deceleration phase (1.4-2s) mostly floated through the domain so that deposition or escape data were not observed.

Considering the initial homogenous distribution of particles injected at the oral inlet, Figure 10 shows particles exiting from different lobes with different colors. Particles leaving the same lobe were closed to each other, thus forming a layered distribution which evolves in time. For example, particles escaped from the LLL lobe are mostly distributed in a central core of the injection (yellow region). The extension of this region reached its maximum value during the first and second injections, then progressively reduced in time.

\section{Discussion}

In the present work, a 3D characteristic model of the airway tree extending from the oral cavity to the $6^{\text {th }}$ generations was developed to evaluate airflow dynamics and aerosol deposition under sedentary breathing physiologically conditions. Morphometric dimensions of the model are consistent with physiological data available in the literature, extracted from invitro preparation of lung casts combined with CT technique. The model was validated by comparing both airflow and pressure distribution through the lobes with their physiological values reported in the literature $[9,16,44,45]$.

Highly unstable phenomena were also correctly captured by the model. The laryngeal jet effect propagates downstream of the trachea (Figures 4-6) with secondary two-vortex structures, proving that the reduced-CSA glottis is the dominant site of production of separation zones, $[14,15,18]$, then the vortex rapidly dissipates downstream the trachea down to the $1^{\text {st }}$ bifurcation. 


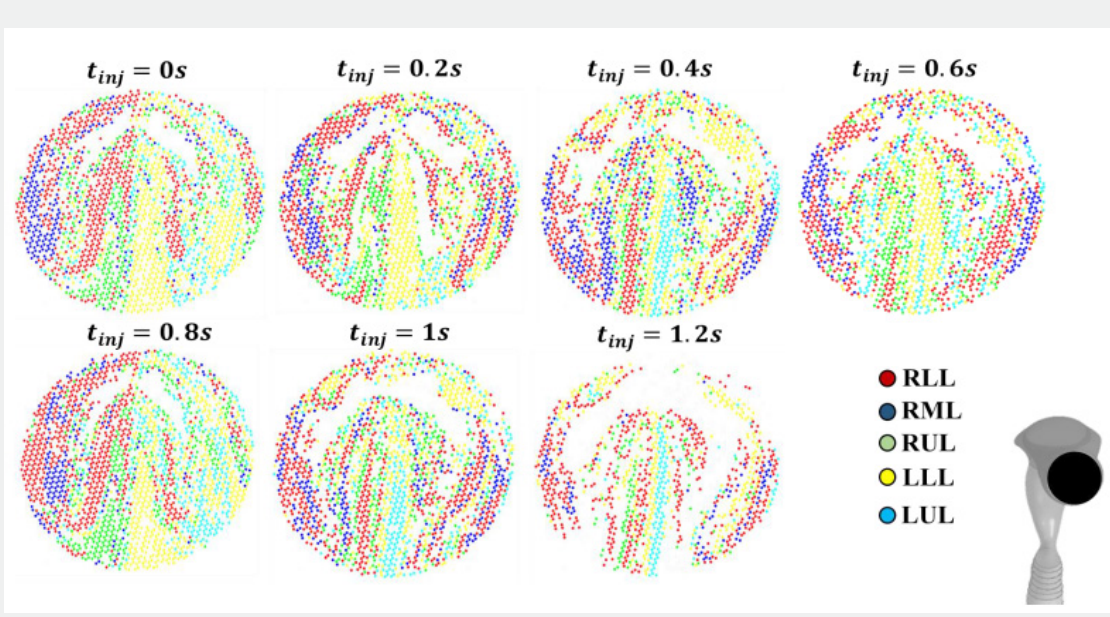

Figure 10: Initial distribution (oral inlet) of $7 \mu \mathrm{m}$ aerosol escaped at different times; different colors indicate the different lobes where aerosol exits, while blank regions represent those deposited or still suspended inside the model.

These results confirm the morphometric accuracy of our model and the importance of including anatomic details like the curvature of the upper airways, the curvatures of bronchi and their branching angles. The unsteady model also proved to be realistic in terms of particle deposition analyses. DE of particles inhaled under sedentary breathing conditions (6 L/ min) showed good agreement with results from previous studies, $[15,19,27,28,30,34]$. Best fitting was provided for $7 \mu \mathrm{m}$ particles, especially at the $1^{\text {st }}$ and $2^{\text {nd }}$ bifurcation Figure 8 . Deposition in trachea shows to be less than how expected from other studies. This trend may be related to the presence of cartilaginous rings features on the trachea walls that acts on the formation of boundary layer.

Total deposition in unsteady conditions is higher than the results obtained in steady: such result points out the necessity to consider unsteady simulation to get a better description of the phenomenon.

Concerning the deposition of $7 \mu \mathrm{m}$ particles inhaled during a complete physiological breathing cycle the sinusoidal airflow pattern showed a major effect on particles behavior. The inhalation of the same particles distribution at different time points led to different results in terms of both regional and total DE. The acceleration phase $(0.6-1 \mathrm{~s})$ proved to be the more efficient, especially on the laryngeal and $3^{\text {rd }}$ bifurcations walls. Furthermore, considering the complete breathing cycle, deposition of particles inhaled in a transient regime was enhanced by $4 \%$ with respect to the constant airflow (steady) situation Figure 9c. The improved deposition at bifurcation walls can be used to enhance the efficacy of therapeutic treatments in these regions.

Concerning particle trajectories, the distribution shown in Figure 10 indicates that injections during the first phases of the breathing cycle (0-0.2s) drive aerosol to deep regions, so that particles leave the model. The number of particles escaped from the lower lobes (RLL, LLL) is larger than that of the particles leaving the upper and medium lobes, but there is no link between the initial position and the lobe that they escape from. Although it is impossible to predict the trajectories of particles based on their initial position, a high percentage of particles escaped could indicate an increased number of particles deposited at terminal bronchioles walls. In this manner, the efficacy of the aerosol treatment could be extended down to deeper bronchioles of the human airways.

About the modelling approach, the description of particles/ droplets deposition, in conditions of laminar or quasi- transitional flow, is an issue common to a number of technical fields. Due to these heterogeneities of applications, it is not unusual to find different numerical settings, derived and dedicated to the geometrical and fluid dynamics conditions under investigation, but non-directly comparable one to each other. About this, in the field of T-junction fluid dynamics, the particles deposition on surfaces can be described by a different $\mathrm{St}$ formulation that represents the ratio of particle inertia to the fluid drag force [47]. Such a representation may fit better to predict particles deposition in bronchi, offering the same trend but higher values of St than the one considered in this paper. On the other hand, such results would not be directly comparable to data available in the reference literature. Over the validation of a model and the comparison of new morphological and physiological conditions, further investigations on adjoining modelling solutions may consolidate the knowledge and the prediction of particles/droplets deposition in bronchi.

Our results show the importance of including the transient behavior of a physiologically breathing cycle to evaluate particle trajectories and deposition under sedentary conditions. Constant airflow simulations underestimate both local and total particle DE. Our model could be used to assess the efficacy of therapeutic aerosol treatment in different regions of human airways down to the 6th generations. Its applicability could be extended to different breathing conditions (i.e. light breathing, heavy breathing) and to different particle types. 


\section{SM1}

LungSim - Multiscale simulation of human lung - https://simtk.org/projects/lungsim

"The simulation software in this repository is a system engineered representation of the human lung based on multiscale imaging (Multidetector CT, micro-CT, microscopy). It allows to investigate dimensions and heterogeneities in geometric branching patterns, gas supply and diffusion capacities. The research being conducted with this tool provides insight into the evolutionary shaped design of the respiratory system, physical optimality and robustness against perturbations caused by the aging process or disease.

LungSim v1.2 is a self-installable file for MS Windows including a demonstration dataset. The models are part of the academic output of the researchers cited and should be referred to as such. The project received funding by DFG and NIH-NIBIB. Developers included in the various phases of LungSim's history are Andreas Schmidt, Thorsten Denhard, Stephan Zidowitz, Saloni Shah, Viraj Shah and Uday K Thummalapalli.

\section{SM2}

Our approach was based on the Octree-based method and Laplace smoothing to generate a high resolution surface mesh, while the volumetric elements were subsequently created with the Delaunay algorithm. Different grids consisting of 8,714,823 (Mesh 1), 9,826,620 (Mesh 2) and 10,496,004 (Mesh 3) cells were created to evaluate the grid size sensitivity for the flow solution.

A boundary layer size sensitivity was also performed to fully capture the highly complex velocity profile near the larynx-glottis walls, discretising the region at a constant distance of $\delta=0.73 \mathrm{~mm}$ from the upper airways and larynx walls with three and ten layers of prismatic elements, respectively.

b)

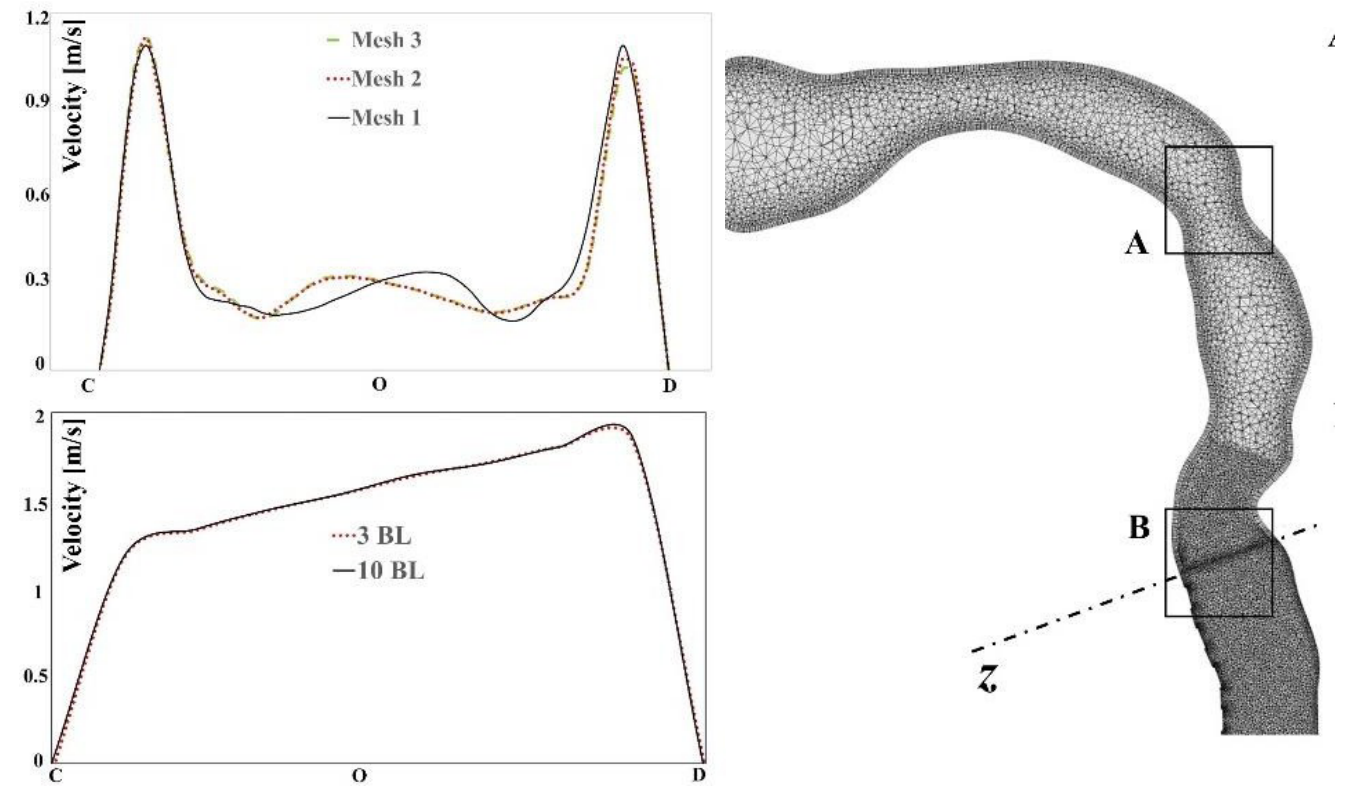

(b) Grid independency results in terms of velocity profile at the two sections A and B in the trachea. BL is the number of elements in the boundary layer.

Figure $3 \mathrm{~b}$ shows the velocity profile over the non-dimensional length in both tracheal and laryngeal sections. Figure 3b indicate that Mesh 1 grid was hardly sufficient to capture airflow fluid dynamics, while the increase of tetrahedral element size up to 10,496,004 cells (Mesh 3) did not change the results in terms of velocity profile in the most critical regions with respect to Mesh 2. Furthermore, three boundary layers proved to be sufficient to fully reconstruct the recirculation zones occurring at laryngeal walls. Hence, Mesh 2 with 9,826,620 tetrahedral elements and three boundary layers of hexahedral ones was adopted to perform the numerical simulations 


\section{SM3 - Preliminary Simulations Steady}

Steady-state simulations were performed to evaluate airflow distribution through the model. A uniform velocity field was applied at the oral inlet with an average value of $0.2211 \mathrm{~m} / \mathrm{s}$. This value corresponds to a minute volume of $6 \mathrm{~L} / \mathrm{min}$, which is representative of sedentary breathing conditions for an average adult male (Guyton, 1977). No-slip boundary conditions were assumed at the airway walls, while constant zero- pressure was prescribed at the 64 outlets of the model. Steady-state solution of the flow field was assumed convergent when the residuals of the governing equations reached values $<10^{\wedge}(-6)$. Flow distribution through the airway model at steady state complies with physiological parameters.

About transient simulations: time step size was set to $5 \mathrm{~ms}$ and the maximum Fluent Courant number was 20 . Transient solution was assumed convergent at each time step when the residuals of the governing equations reached values $<10^{\wedge}(-4)$, in accordance with other studies found in literature [14].

\section{SM4}

The motion of spherical liquid particles suspended in air is governed by Newton's Second Law:

$$
\frac{d \overrightarrow{u_{p, l}}}{d t}=\sum \overrightarrow{F_{p}}
$$

where $u_{p, l}$ is the velocity of the $i$ particle and $F p$ the force acting on it

Considering small particles, Reynolds numbers $R e_{p}=\frac{\rho d_{p}\left|u_{p, i}-u_{i}\right|}{\mu}<<1$, a large density ratio $\rho p$ p $\gg$ and particle diameter $\mathrm{d}_{\mathrm{p}}>1 \mu \mathrm{m}$, most of the known particle forces other than drag force can be neglected [32], thus Eq. (6) can be simplified as follows:

$$
\frac{d \overrightarrow{u_{p, l}}}{d t}=F_{D}\left(\overrightarrow{u_{l}}-\overrightarrow{u_{p, l}}\right)
$$

where $u_{p, i}$ and $u_{i}$ are the components of particle and fluid velocity in the $i-t h$ position, respectively. For spherical particles, the drag force per unit mass is defined as:

$$
F_{D}=\frac{18 \mu}{\rho_{p} d_{p}^{2} c_{c}} \frac{C_{D} \operatorname{Re}_{p}}{24} \text { (8) }
$$

where $C_{D}$ is the drag coefficient defined by Morsi and Alexander (Morsi, 1972), $d_{p}$ is the particle diameter and $C_{c}$ is the Cunningham slip correction factor defined by Hinds et al. [31]. With particle Reynolds numbers $\operatorname{Re}_{\mathrm{p}}<1$ and very small Stokes numbers (St $<$ 0.25), several authors found that particle motion can be basically achieved with a first-order correction to fluid element motion equation [5,6,32]. For example, Comer observed that the largest influence of the Cunningham slip correction factor $C_{c}$ was a $2 \%$ change in particle $\mathrm{DE}$ at $\mathrm{St}=0.24$ (Comer, $\mathrm{PhD}$ thesis publication). In this sense, $C_{c}$ is used for particles in the nanometric range, where the primary phase cannot be considered continuous [42]. Furthermore, the correction to Stokes law for non-rigid spheres as water droplets is generally insignificant ([31,42]; Fluent Guide, Fluent 16, Ansys Inc.).

\section{SM5}

Table 1: Deposition efficiency (DE) of 3-25 $\mu$ m particles injected under steady conditions (Q=6 L/min).

\begin{tabular}{|c|c|c|c|c|}
\hline \multirow{2}{*}{ Region } & \multicolumn{4}{|c|}{ Particle diameter $[\boldsymbol{\mu m}]$} \\
\cline { 2 - 5 } & $\mathbf{3}$ & $\mathbf{7}$ & $\mathbf{1 0}$ & $\mathbf{2 5}$ \\
\hline Pharynx & $0.33 \%$ & $0.29 \%$ & $0.40 \%$ & $3.86 \%$ \\
\hline Larynx & $\mathbf{5 . 2 9 \%}$ & $\mathbf{6 . 2 9} \%$ & $\mathbf{8 . 7 5 \%}$ & $\mathbf{5 3 . 2 4 \%}$ \\
\hline Trachea & $0.77 \%$ & $1.07 \%$ & $2.43 \%$ & $14.34 \%$ \\
\hline $1^{\text {st } \text { bifurcation }}$ & $1.47 \%$ & $1.43 \%$ & $1.51 \%$ & $0.59 \%$ \\
\hline RUL & $0.44 \%$ & $0.40 \%$ & $1.51 \%$ & $7.68 \%$ \\
\hline RLL & $1.88 \%$ & $2.13 \%$ & $3.71 \%$ & $5.74 \%$ \\
\hline LUL & $0.29 \%$ & $1.03 \%$ & $1.14 \%$ & $1.76 \%$ \\
\hline LLL & $0.99 \%$ & $1.80 \%$ & $3.05 \%$ & $4.04 \%$ \\
\hline Total DE & $\mathbf{1 1 . 4 7 \%}$ & $\mathbf{1 4 . 4 5 \%}$ & $\mathbf{2 2 . 5 1 \%}$ & $\mathbf{9 1 . 2 5 \%}$ \\
\hline
\end{tabular}

RUL and RLL are the right upper and lower lobes, LUL and LLL are the left upper and lower ones. Grey cells represent the maximum DE of each region of the model, bold font highlights the maximum DE for various particle diameters. 


\section{Conclusion}

The present 3D model of the respiratory tree contains characteristic geometric details of the oral cavity, pharynx, larynx and TB tree down to the $6^{\text {th }}$ generations. Morphologic and morphometric features guarantee a physiological airflow distribution through pulmonary lobes and realistic TB pressure drops for an average adult male patient.

Transient simulation of a complete breathing cycle allowed us to describe highly unstable phenomena like the formation of a laryngeal jet and recirculation patterns and investigate the resulting aerosol deposition. Aerosol DE was analyzed at various injection times and compared with those observed under constant airflow rates. The number of particles leaving each lobe was also assessed. Our results show the importance of including a transient physiologically breathing cycle on aerosol deposition analyses. Furthermore, the results indicate that there not relation between the position of the inhalation across the mouth and the deposition lobe, because the vorticity in the glottides and trachea sections mix the trajectories on the transversal plane.

In conclusion, our 3D model can be used to correctly analyze airflow pattern and aerosol behavior for an average adult male. It could be modified/scaled so as to allow analysis of different breathing pattern (i.e. light breathing, heavy breathing) or different subjects (i.e. average paediatric or elderly) or pathological airways conditions. The proposed model was developed to evaluate airflow dynamics for an average adult male patient under sedentary breathing conditions. After geometric adaption the model could be extended to explore airflow dynamics following morphological changes (e.g. due to age and sex) as well as under pathological conditions.

Future research directions could include the extension of the model to deeper generations in order to evaluate $\mathrm{DE}$ and thus aerosol efficacy further down to the bronchiole regions. Computational simulations tools have proved to be able to show flows and particles depositions, to be able to assemble data from several geometrical features and time instant into a usable frames, as tools for the design of protocols and devices for aerosol administration. Deeper anatomical structures, not included in this model, could be an improvement only if the reliability of results is preserved: the deformability of further bifurcation structures may affect the definitions of boundaries along the breath cycle so it should be considered in the definition of a more extended model.

\section{Acknowledgments}

This work was partly supported by Fondazione per la Ricerca Scientifica Termale (FoRST, Rome, Italy). The authors gratefully acknowledge helpful discussions with Prof. Luca Cortelezzi, Politecnico di Milano, on the numerical approach on droplet deposition.

\section{References}

1. Wang C (2005) Inhaled Particles. Elsevier.

2. Islam MS, Paul G, Ong HX, Young PM, Gu YT, et al. (2010) A Review of Respiratory Anatomical Development, Air Flow Characterization and Particle Deposition. Int J Environ Res Public Health 17(2): 380.

3. Walenga RL, Tian G, Longest PW (2013) Development of characteristic upper tracheobronchial airway models for testing pharmaceutical aerosol delivery. J Biomech Eng 135(9): 91010.

4. Balásházy U, Hofmann W (1993) Particle deposition in airway bifurcations-I. Inspiratory flow. Journal of Aerosol Science 24(6): 745772 .

5. Comer JK, Kleinstreuer C, Hyun S, Kim CS (2000) Aerosol Transport and Deposition in Sequentially Bifurcating Airways. J Biomech Eng (2): 152-158.

6. Zhang Z, Kleinstreuer C, Kim CS (2001) Flow Structure and Particle Transport in a Triple Bifurcation Airway Model. J Fluids Eng 123(2): 320-330.

7. Kim YH, Tong ZB, Chan HK, Yang RY (2019) CFD modelling of air and particle flows in different airway models. Journal of Aerosol Science 134: 14-28.

8. Schmidt A, Zidowitz S, Kriete A, Denhard T, Krass S, et al. (2004) A digital reference model of the human bronchial tree. Comput Med Imaging Graph 28(4): 203-211.

9. Gemci T, Ponyavin V, Chen Y, Chen H, Collins R (2008) Computational model of airflow in upper 17 generations of human respiratory tract. Journal of Biomechanics 41(9): 2047-2054.

10. Farghadan A, Coletti F, Arzani A (2019) Topological analysis of particle transport in lung airways: Predicting particle source and destination. Computers in Biology and Medicine 115: 103497.

11. Cheng K, Cheng Y, Yeh H, Swift DL (1997) Measurements of Airway Dimensions and Calculation of Mass Transfer Characteristics of the Human Oral Passage. J Biomech Eng 119(4): 476-482.

12. Stapleton KW, Guentsch E, Hoskinson MK, Finlay WH (2000) On the suitability of $\mathrm{k}-\varepsilon$ turbulence modeling for aerosol deposition in the mouth and throat: a comparison with experiment. Journal of Aerosol Science 31(6): 739-749.

13. Xi J, Longest PW (2007) Transport and deposition of micro-aerosols in realistic and simplified models of the oral airway. Ann Biomed Eng 35(4): 560-581.

14. Elcner J, Lizal F, Jedelsky J, Jicha M, Chovancova M (2016) Numerical investigation of inspiratory airflow in a realistic model of the human tracheobronchial airways and a comparison with experimental results. Biomech Model Mechanobiol 15(2): 447-469.

15. Lambert AR, O’Shaughnessy P, Tawhai MH, Hoffman EA, Lin C (2011) Regional deposition of particles in an image-based airway model: large-eddy simulation and left-right lung ventilation asymmetry. Aerosol Sci Technol 45(1): 11-25.

16. Tian G, Longest PW, Su G, Walenga RL, Hindle M (2011) Development of a stochastic individual path (SIP) model for predicting the tracheobronchial deposition of pharmaceutical aerosols: Effects of transient inhalation and sampling the airways. Journal of Aerosol Science 42(11): 781-799.

17. Yousefi M, Pourmehran O, Gorji-Bandpy M, Inthavong K, Yeo L, et al. (2017) CFD simulation of aerosol delivery to a human lung via surface acoustic wave nebulization. Biomech Model Mechanobiol 16(6): 20352050. 
18. Calmet H, Gambaruto AM, Bates AJ, Vázquez M, Houzeaux G, et al. (2016) Large-scale CFD simulations of the transitional and turbulent regime for the large human airways during rapid inhalation. Computers in Biology and Medicine 69: 166-180.

19. Rahimi-Gorji M, Gorji TB, Gorji-Bandpy M (2016) Details of regional particle deposition and airflow structures in a realistic model of human tracheobronchial airways: two-phase flow simulation. Comput Biol Med 74: 1-17.

20. Das P, Nof E, Amirav I, Kassinos SC, Sznitman J (2018) Targeting inhaled aerosol delivery to upper airways in children: Insight from computational fluid dynamics (CFD). PLoS One 13(11).

21. Weibel ER (1963) Morphometry of the Human Lung. Berlin Heidelberg: Springer-Verlag.

22. Balásházy I, Hofmann W (1995) Deposition of aerosols in asymmetric airway bifurcations. Journal of Aerosol Science 26(2): 273-292.

23. Heistracher T, Hofmann W (1995) Physiologically realistic models of bronchial airway bifurcations. Journal of Aerosol Science 26(3): $497-$ 509

24. Koullapis PG, Hofemeier P, Sznitman J, Kassinos SC (2018) An efficient computational fluid-particle dynamics method to predict deposition in a simplified approximation of the deep lung. Eur J Pharm Sci 113 132-144.

25. Islam MS, Saha SC, Sauret E, Gemci T, Yang IA, et al. (2017) Ultrafine particle transport and deposition in a large scale 17-generation lung model. J Biomech 64: 16-25.

26. Finlay WH (2001) The Mechanics of Inhaled Pharmaceutical Aerosols.

27. Zhou Y, Cheng Y (2005) Particle Deposition in a Cast of Human Tracheobronchial Airways. Aerosol Science and Technology 39(6): 492-500.

28. Cheng Y, Zhou Y, Chen BT (1999) Particle Deposition in a Cast of Human Oral Airways. Aerosol Science and Technology 31(4): 286-300.

29. Schlesinger RB, Bohning DE, Chan TL, Lippmann M (1977) Particle deposition in a hollow cast of the human tracheobronchial tree. Journal of Aerosol Science 8(6): 429-445.

30. Chan TL, Lippmann M (1980) Experimental measurements and empirical modelling of the regional deposition of inhaled particles in humans. Am Ind Hyg Assoc J 41(6): 399-409.

31. Hinds WC (1999) Aerosol Technology: Properties, Behavior, and Measurement of Airborne Particles.

32. Zhang Z, Kleinstreuer C, Kim CS (2002) Computational analysis of micron-particle deposition in a human triple bifurcation airway model. Comput Methods Biomech Biomed Engin 5(2): 135-147.
33. Cheng YS (2003) Aerosol Deposition in the Extrathoracic Region. Aerosol Sci Technol 37(8): 659-671.

34. Schlesinger RB, Bohning DE, Chan TL, Lippmann M (1977) Particle deposition in a hollow cast of the human tracheobronchial tree. Journal of Aerosol Science 8(6): 429-445.

35. Kim CS, Fisher DM (1999) Deposition Characteristics of Aerosol Particles in Sequentially Bifurcating Airway Models. Aerosol Science and Technology 31(2-3): 198-220.

36. Gurman JL, Lioy PJ, Lippmann M, Schlesinger RB (1984) Particle Deposition in Replicate Casts of the Human Upper Tracheobronchial Tree Under Constant and Cyclic Inspiratory Flow. II. Experimental Model. Aerosol Science and Technology 3(3): 253-257.

37. Stahlhofen W, Gebhart J, Heyder J (1980) Experimental determination of the regional deposition of aerosol particles in the human respiratory tract. Am Ind Hyg Assoc J 41(6): 385-398a.

38. Kolanjiyil AV, Kleinstreuer C (2017) Computational analysis of aerosoldynamics in a human whole-lung airway model. Journal of Aerosol Science 114: 301-316

39. Russo J, Robinson R, Oldham MJ (2008) Effects of cartilage rings on airflow and particle deposition in the trachea and main bronchi. Med Eng Phys 30(5): 581-589.

40. Randestad A, Lindholm CE, Fabian P (2000) Dimensions of the cricoid cartilage and the trachea. Laryngoscope 110(11): 1957-1961.

41. Bairati A (1974) Trattato di anatomia umana, morfologia generale e sistematica dell'uomo. : Minerva Medica.

42. Tu J, Inthavong K, Ahmadi G (2013) Computational Fluid and Particle Dynamics in the Human Respiratory System. Springer

43. Tian G, Hindle M, Lee S, Longest PW (2015) Validating CFD Predictions of Pharmaceutical Aerosol Deposition with In Vivo Data. Pharm Res 32(10): 3170-3187.

44. Horsfield K, Dart G, Olson DE, Filley GF, Cumming G (1971) Models of the human bronchial tree. J Appl Physiol 31(2): 207-217.

45. Cohen BS, Sussman RG, Lippmann M (1990) Ultrafine Particle Deposition in a Human Tracheobronchial Cast. Aerosol Science and Technology 12(4): 1082-1091.

46. Zhang Z, Kleinstreuer C, Kim CS (2008) Airflow and Nanoparticle Deposition in a 16-Generation Tracheobronchial Airway Model. Ann Biomed Eng 36(12): 2095-2110.

47. Vigolo D, Griffiths IM, Radl S, Stone HA (2013) An experimental and theoretical investigation of particle-wall impacts in a T-junction. Journal of Fluid Mechanics 727: 236-255.

This work is licensed under Creative Commons Attribution 4.0 License

DOI: 10.19080/CTBEB 2020.19.556023

\section{Your next submission with Juniper Publishers} will reach you the below assets

- Quality Editorial service

- Swift Peer Review

- Reprints availability

- E-prints Service

- Manuscript Podcast for convenient understanding

- Global attainment for your research

- Manuscript accessibility in different formats

( Pdf, E-pub, Full Text, Audio)

- Unceasing customer service

Track the below URL for one-step submission

https://juniperpublishers.com/online-submission.php 